\title{
Convergence Study of Biogeography Based Optimization
}

\author{
D. K. Mishra ${ }^{1}$, Vikas Shinde ${ }^{2}$, Kamal Wadhwa ${ }^{3}$ and Sanjay Chaudhary ${ }^{4}$ \\ ${ }^{1 \& 4}$ Department of Mathematics, Government Narmada P.G. College, Hoshangabad, Madhya Pradesh, India \\ ${ }^{3}$ Department of Mathematics, Government P.G. College, Pipariya, Madhya Pradesh, India \\ ${ }^{2}$ Department of Applied Mathematics, Madhav Institute of Technology \& Science, Gwalior, Madhya Pradesh, India \\ Email: dilipmishra3826@gmail.com, v_p_shinde@rediffmail.com
}

\begin{abstract}
Biogeography based optimization BBO is a progressive algorithm. It is induced by Biogeography. BBO is more powerful algorithm among the biology based optimization methods. In this paper examines the convergence of BBO algorithm on some fitness functions. BBO algorithm handles the best solution from one off spring to the next converges to the universal optimum. The convergence rate evaluate of $\mathrm{BBO}$ algorithm by simulation for some fitness function. A set of 12 standard benchmark function performance of convergence is studied by BBO algorithm.

Keywords: BBO Algorithm, Migration, Mutation, Emigration
\end{abstract}

\section{INTRODUCTION}

BBO relates a new type of evolutionary algorithm. A mathematical model of biogeography depicts the immigration and emigration of biological organism. It was first adduced in 2008[3]. BBO replicas after immigration and emigration of biological organisms between odor. It applies to the fitness of each organism result to obtain its immigration and emigration rate. The immigration rate obtains then a biological organism solution is to change its decision variables. It has showed better performance on many unconstrained and constrained benchmark functions. An optimization problem is a real world optimization problem. Some mathematical benchmark functions have been used to find Evolutionary Algorithm convergence. Mathematical modeling is based on biogeography based optimization, how the breed transfer from one place to another place. How new breeds grow and how destructed the breeds. There are two types of migration in BBO, like that emigration and imigration both are influenced by many factors e.g., distance of isle to the nearby neighbor, dimension of the isle, Habitat suitability index (HSI) etc. HSI depends on many elements like that vegetation, atmosphere, rainfall etc. These elements support the implicitness of breeds in a (habitat) provenance. Provenances are favorable for the living place of biological breeds. They will be advanced HSI [1]. Many number of breeds will be engaged by high HSI to Habitat.

In Breeds distribution, high HSI habitats are more consistent than low HSI habitats. There are so many opportunities on high HSI Isle for emigrating to near habitats. Biogeography is type of dispensation of breeds and it is similar to ordinary problem solutions. Suppose some relevant issues and their solutions are presented like that in economics, business, sports, medical science etc. A perfect rectification is similar to an isle with a high HSI and an imperfect rectification describes an isle with a low HSI. Many researchers have been evaluated and examined the performance of $\mathrm{BBO}$ on several benchmark functions. Guo and $\mathrm{Yu}$ [2] Considered optimization algorithm converge to the universal optimum, When the population limit tends to infinity then candidate solution exist for at least one value, which provide the global solution using various mathematical tools of the optimization problem. Simon [3] considered the natural biogeography and its related mathematical tools, how to solve optimization problem.

$\mathrm{Ma}$ and Simon [4] BBO is a new approach of EA for getting best performance on various unconstrained and constrained benchmark functions have been studied. Ma [5] demonstrated the migration models in BBO and explores the execution through different benchmark functions. Simon et.al [6] derived the proportion of each individual in the population for a given optimization problem using theory rather than simulation dynamic system model for biogeography-based optimization (BBO). Ilhem et al., [7] Evaluated the result for given objective function for the constrained optimization i.e. inequality and equality problems by $\mathrm{BBO}$. Ma et al., [8] described $\mathrm{BBO}$ for multi objective optimization problem wherein proposed algorithm is used to non dominated sorting approach to improve the convergence efficiency. Hordri [9] investigated the performance of BBO, GA and PSO for convergence. Feng et al., [10] proposed modified Biogeography-Based optimization with Local Search Mechanism for migration operator in $\mathrm{BBO}$, through it more information can be extracted from other habitats. Guo et al., [11] explained the effect of migration rates on $\mathrm{BBO}$; these are useful for designing of migration model.

Ma et al., [12] Analyzed the Biogeography-Based optimization for Binary Problems provide the best candidate in the population from one generation to the next which converges to the global optimum solution. Golafshani [13] explored the impact of the Biogeography Based Programming for several benchmark functions to solve the problems. Weian et al., [14] Investigated migration models for Multi-Objective Problems (MOPs) using BBO. Ma and Simon [15] considered a BBO evolutionary algorithm papers since last 10 years wherein they summarized and organized the literature. Khademi et al., [16] considering the significant and expanding research of $\mathrm{BBO}$ and its applications in different domain. 
We organized the paper as follows. In section II, described the biogeography-based optimization (BBO) algorithm. Convergence study of biogeography-based optimization (BBO) algorithm is proposed, in section, III. Finally conclusion is drawn in section IV.

\section{BIOGEOGRAPHY BASED OPTIMIZATION (BBO)}

As observed the Biogeography based optimization algorithm which is introduced by Dan Simon. It is a new appearing population based algorithm. In BBO algorithm, feasible result depends on the habitat and their characteristics . Their characteristics described with merits are called merit/suitability index variables (SIVs). In BBO, there are some common features with other biological based algorithms, like PSO, GAs and BBO. If each solution is desirable then it is known as habitat suitability index variables (SIVs).

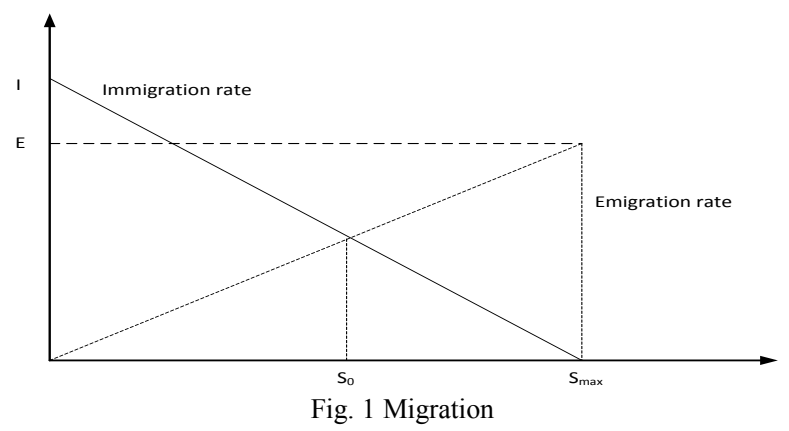

A. Migration

Migration is random operator. It is studied to enhance the candidate solution Fi. For every characteristics of a given candidate solution $f_{i}$, the candidate solution's immigration rate $\lambda_{\mathrm{k}}$ is used to randomly decide whether to immigrate or not. If the possibility immigration is there, then the emigration candidate solution $f_{j}$ is randomly chosen based on the emigration rate $\mu_{\mathrm{j}}$, it is given as

$$
f_{i}(r) \leftarrow f_{j}(r)
$$

where $r$ is a symbol of candidate solution. In BBO, every candidate solution $f_{i}$ has its own immigration rate $\lambda_{\mathrm{k}}$ and emigration rate $\mu_{\mathrm{k}}$. A valid result has relatively high $\mu$ and low $\lambda$, however the converse is true for a weak candidate solution. Immigration rate $\lambda_{\mathrm{k}}$ and emigration rate $\mu_{\mathrm{k}}$ of the candidate solution can be achieved by using (1)

$$
\begin{gathered}
\lambda_{k}=1-\text { fitness }\left(f_{i}\right) \\
\mu_{k}=\text { fitness }\left(f_{i}\right)
\end{gathered}
$$

where fitness indicates candidate solution of fitness value. It's range is $[0,1]$ The probabilities of immigrating to $f_{i}$ and of emigrating from $f_{i}$ are obtained as

$$
\begin{aligned}
& \operatorname{Pr}\left(\text { immigration to } f_{i}\right)=\lambda_{k} \\
& \operatorname{Pr}\left(\text { emmigration from } f_{i}\right)=\frac{\mu_{k}}{\sum_{j=1}^{N} \mu_{j}}
\end{aligned}
$$

where $\mathrm{N}$ is population size.

\section{B. Mutation}

Mutation is random operator that randomly improves a candidate solution characteristic; the objective of mutation is to enhance diversity among the population. Each population candidate is related randomly, which denotes the presumption that it was required to exist as a result to the given problem. Very high HSI results and very low HSI results are not possible to equal improbable. Medium HSI results are expected to be probable. In a case result $\mathrm{R}$ has a low probability $\mathrm{P}_{\mathrm{R}}$, and then astoundingly it exists as a result. It seems to mutate to some other result. Other than, a solution with a high probability has less chance to mutate to different results. It is denoted as mutation rate $\mathrm{m}$.

$$
m(R)=m_{\max }\left(\frac{1-P_{R}}{P_{\max }}\right)
$$

Where $\mathrm{M}_{\max }$ is a user defined parameter. Mutation approach makes low HSI solutions likely to mutate which gives them a chance of improving. It also provides high HSI solutions likely to mutate which gives them a chance of improving even more than they already have.

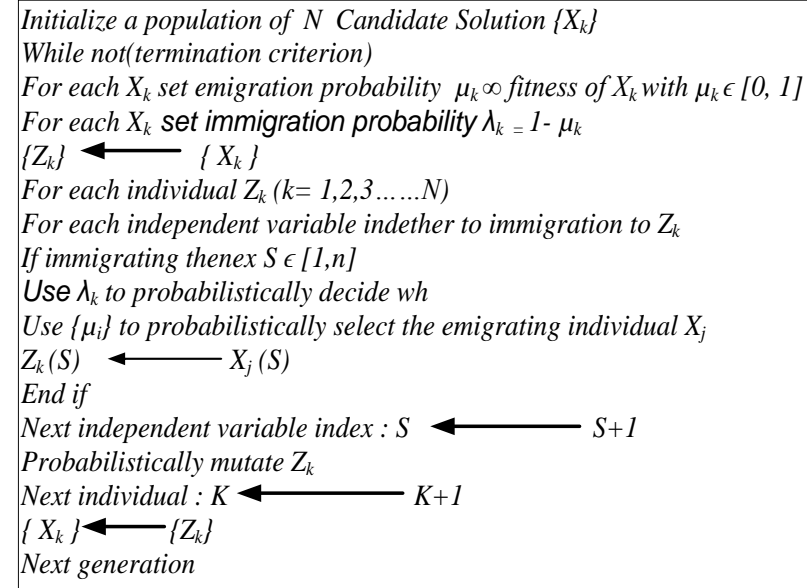

Fig. 2 Mutation

\section{CONVERGENCE OF BBO ALGORITHM}

Different optimization algorithms are local search algorithm out of which meta-heuristic algorithms are appropriate for obtaining global optimization. Many algorithms may be trapped in a local optimum. As we have shown the BBO algorithm has good global convergence property. It can be more significant for global optimization. In order to depict the $\mathrm{BBO}$ algorithm has good convergence for various function. 
TABLE I THE BENCHMARK TEST FUNCTIONS

\begin{tabular}{|c|c|c|c|c|}
\hline Name & Test Function & Nature of Function & $\begin{array}{l}\text { Search } \\
\text { Space }\end{array}$ & $F_{\min }$ \\
\hline Ackley & $f_{1}(x)=-20 e^{-0.02 \sqrt{D^{-1} \sum_{i=1}^{D} x_{i}^{2}}-D^{-1} \sum_{i=1}^{D} \cos \left(2 \pi x_{i}\right)+20+\varepsilon}$ & $\begin{array}{l}\text { Continuous, } \\
\text { Differentiable, Non- } \\
\text { Separable, Scalable, } \\
\text { Multi-Model }\end{array}$ & {$[-32,32]$} & 0 \\
\hline Beale & $f_{2}(x)=\left(1.5-x_{1}+x_{1} x_{2}\right)^{2}+\left(2.25-x_{1}+x_{1} x_{2}^{2}\right)^{2}++\left(2.625-x_{1}+x_{1} x_{2}^{3}\right)^{2}$ & $\begin{array}{l}\text { Continuous, } \\
\text { Differentiable, Non- } \\
\text { Separable, Non- } \\
\text { Scalable, Multi-Model }\end{array}$ & {$[-4.5,4.5]$} & 0 \\
\hline Booth & $f_{2}(x)=\left(x_{1}+2 x_{2}-7\right)^{2}+\left(2 x_{1}+x_{2}-5\right)^{2}$ & $\begin{array}{l}\text { Continuous, } \\
\text { Differentiable, Non- } \\
\text { Separable, Non- } \\
\text { Scalable, Uni-Model }\end{array}$ & {$[-10,10]$} & 0 \\
\hline $\begin{array}{l}\text { Carrom } \\
\text { table }\end{array}$ & $f_{4}(x)=-\left[\left\{\operatorname{Cos}\left(x_{1}\right) \operatorname{Cos}\left(x_{2}\right) e^{\mid 1-\left(x_{1}^{2}+x_{2}^{2}\right)^{\frac{0.5}{\pi}}} \mid\right\}^{2}\right] / 30$ & $\begin{array}{l}\text { Continuous, } \\
\text { Differentiable, } \\
\text { Separable, Scalable, } \\
\text { Multi-Model }\end{array}$ & {$[-10,10]$} & 24.1566 \\
\hline $\begin{array}{l}\text { Crowned } \\
\text { cross }\end{array}$ & $f_{5}(x)=0.0001\left[\mid \sin \left(x_{1}\right) \operatorname{Sin}\left(x_{2}\right) e^{\left.\| 100-\left[\left(x_{1}^{2}+x_{2}^{2}\right)^{0.5}\right] / n||\right]} / 1\right.$ & $\begin{array}{l}\text { Continuous, } \\
\text { Differentiable, } \\
\text { Separable, Scalable, } \\
\text { Multi-Model }\end{array}$ & {$[-10,10]$} & 0.0001 \\
\hline $\begin{array}{l}\text { Corss in } \\
\text { Tray }\end{array}$ & $f_{6}(x)=-0.0001\left[\left|\sin \left(x_{1}\right) \sin \left(x_{2}\right) e^{\mid 100-\left[\left(x_{1}^{2}+x_{2}^{2}\right)^{\frac{0.5}{\pi}}\right.} \|\right| \mid+1\right.$ & $\begin{array}{l}\text { Continuous, Non- } \\
\text { Separable, Non- } \\
\text { Scalable, Multi-Model }\end{array}$ & {$[-10,10]$} & -2.0626 \\
\hline Easom & $f_{7}(x)=-\operatorname{Cos}\left(x_{1}\right) \operatorname{Cos}\left(x_{2}\right) \exp$ & $\begin{array}{l}\text { Continuous, } \\
\text { Differentiable, } \\
\text { Separable, Non- } \\
\text { Scalable, Multi-Model }\end{array}$ & {$[-100,100]$} & -1 \\
\hline Powell & $f_{g}(x)$ & $\begin{array}{l}\text { Continuous, Non- } \\
\text { Differentiable, } \\
\text { Separable, Uni-Model, } \\
\text { Convex dimensional }\end{array}$ & {$[-1,1]$} & 0 \\
\hline $\begin{array}{l}\text { Pen } \\
\text { Holder }\end{array}$ & $\left(x_{2}\right)_{e}\left|1-\left(x_{1}^{2}+x_{2}^{2}\right)^{\frac{0.5}{\pi}}\right|||^{-1}$ & $\begin{array}{l}\text { Continuous, } \\
\text { Differentiable, Non- } \\
\text { Separable, , Non- } \\
\text { Scalable, Multi-Model }\end{array}$ & {$[-11,11]$} & $\begin{array}{c}- \\
0.96354\end{array}$ \\
\hline Rastigin & $f_{10}(x)=10 d+\sum_{i=1}^{\infty}\left[x_{i}^{2}\right.$ & $\begin{array}{l}\text { Continuous, } \\
\text { Differentiable, } \\
\text { Separable, Scalable, } \\
\text { Multi-Model }\end{array}$ & {$[-5.12,5.12]$} & 0 \\
\hline Schwefel & $f_{11}(x)=\sum_{i=1}\left|x_{i}\right|$ & $\begin{array}{l}\text { Continuous, Non- } \\
\text { Differentiable, } \\
\text { Separable, Scalable, } \\
\text { Uni-Model }\end{array}$ & {$[-10,10]$} & 0 \\
\hline Sphere & $f_{12}(x)=\sum_{i=1}^{n} x_{i}^{2}$ & $\begin{array}{l}\text { Continuous, } \\
\text { Differentiable, } \\
\text { Separable, Scalable, } \\
\text { Multi-Model }\end{array}$ & {$[-100,100]$} & 0 \\
\hline
\end{tabular}


TABLE II THE BENCHMARK TEST FUNCTIONS

\begin{tabular}{|c|c|c|c|c|c|}
\hline $\begin{array}{l}\text { Functions } \\
\text { Name }\end{array}$ & Population Size & $\begin{array}{c}\text { Number of } \\
\text { Iteration }\end{array}$ & Dim. & Best & Global Minima \\
\hline \multirow{3}{*}{ Ackley } & 200 & 1000 & \multirow{3}{*}{2} & 0.000000 & \multirow{3}{*}{0} \\
\hline & 100 & 700 & & 0.00000695 & \\
\hline & 50 & 500 & & 0.0007155 & \\
\hline \multirow{3}{*}{ Beale } & 200 & 1000 & \multirow{3}{*}{2} & 0.00000001 & \multirow{3}{*}{0} \\
\hline & 100 & 700 & & 0.00000004 & \\
\hline & 50 & 500 & & 0.00000288 & \\
\hline \multirow{3}{*}{ Booth } & 200 & 1000 & \multirow{3}{*}{2} & .0001194 & \multirow{3}{*}{0} \\
\hline & 100 & 700 & & 0.000347 & \\
\hline & 50 & 500 & & 0.00001051 & \\
\hline \multirow{3}{*}{ CarromTable } & 200 & 1000 & \multirow{3}{*}{2} & -24.1568 & \multirow{3}{*}{-24.1568} \\
\hline & 100 & 700 & & -24.1568 & \\
\hline & 50 & 500 & & -24.1568 & \\
\hline \multirow{3}{*}{ Crowned Cross } & 200 & 1000 & \multirow{3}{*}{2} & 0.00012072 & \multirow{3}{*}{0.00014} \\
\hline & 100 & 700 & & 0.00011774 & \\
\hline & 50 & 500 & & 0.00015515 & \\
\hline \multirow{3}{*}{ Crossin Tray } & 200 & 1000 & \multirow{3}{*}{2} & -2.0626 & \multirow{3}{*}{-2.0626} \\
\hline & 100 & 700 & & -2.0626 & \\
\hline & 50 & 500 & & -2.0626 & \\
\hline \multirow{3}{*}{ Easom } & 200 & 1000 & \multirow{3}{*}{2} & -1 & \multirow{3}{*}{-1} \\
\hline & 100 & 700 & & -1 & \\
\hline & 50 & 500 & & -1 & \\
\hline \multirow{3}{*}{ Penholder } & 200 & 1000 & \multirow{3}{*}{2} & -0.96353 & \multirow{3}{*}{-0.96353} \\
\hline & 100 & 700 & & -0.96353 & \\
\hline & 50 & 500 & & -0.96353 & \\
\hline \multirow{3}{*}{ Powell } & 200 & 1000 & \multirow{3}{*}{2} & Nan & \multirow{3}{*}{0} \\
\hline & 100 & 700 & & Nan & \\
\hline & 50 & 500 & & Nan & \\
\hline \multirow{3}{*}{ Rastrigin } & 200 & 1000 & & 0 & \\
\hline & 100 & 700 & 2 & 0 & 0 \\
\hline & 50 & 500 & & 0.0000355 & \\
\hline & 200 & 1000 & & -837.9658 & \\
\hline Schweffel & 100 & 700 & 2 & -837.9658 & 0 \\
\hline & 50 & 500 & & -837.9658 & \\
\hline & 200 & 1000 & & $2.6322 \times 10-12$ & \\
\hline Sphere & 100 & 700 & 2 & $4.6313 \times 10-10$ & 0 \\
\hline & 50 & 500 & & $5.5429 \times 10-8$ & \\
\hline
\end{tabular}




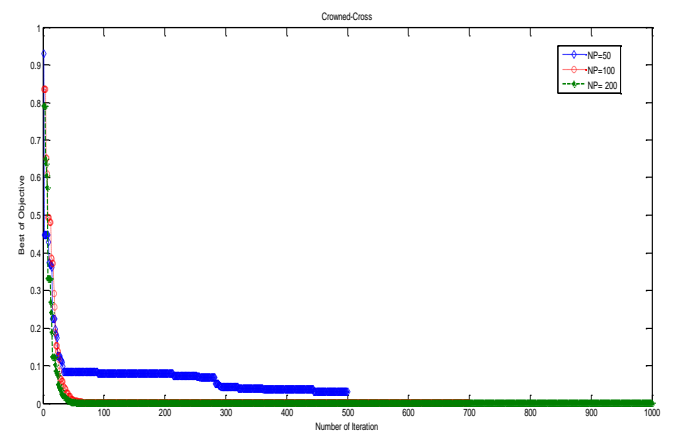

Fiig. 3 Ackley

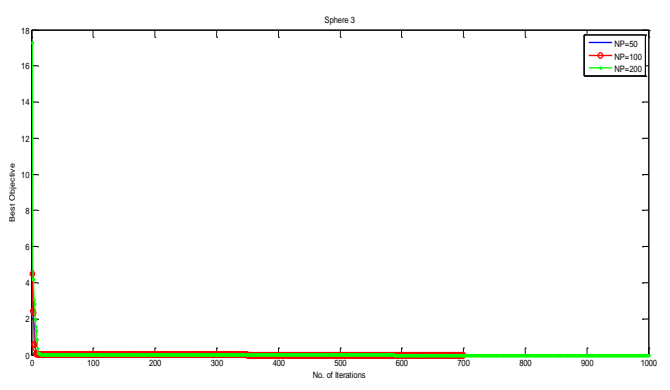

Fig. 5 Booth

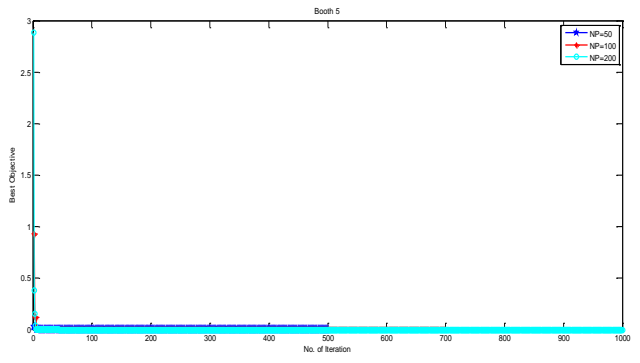

Fig. 6 Carrom Table

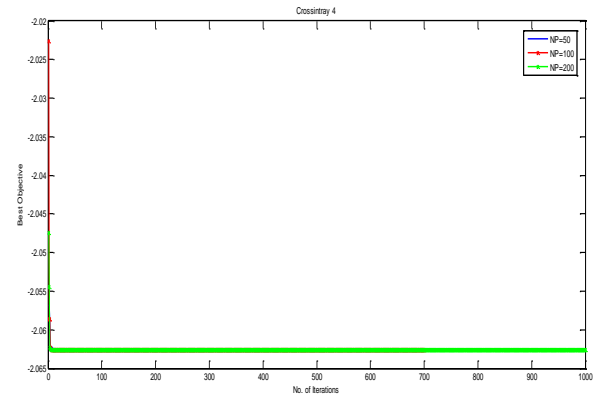

Fig. 7 Crowned Cross

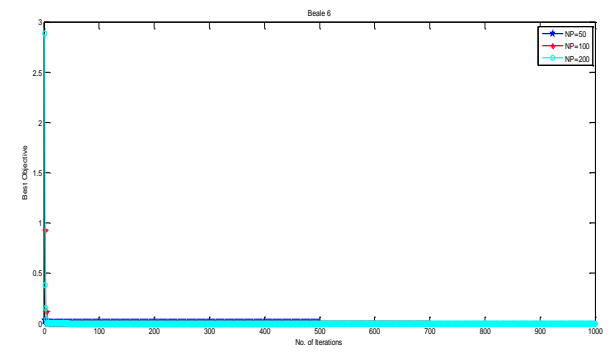

Fig. 8 Crossin Tray

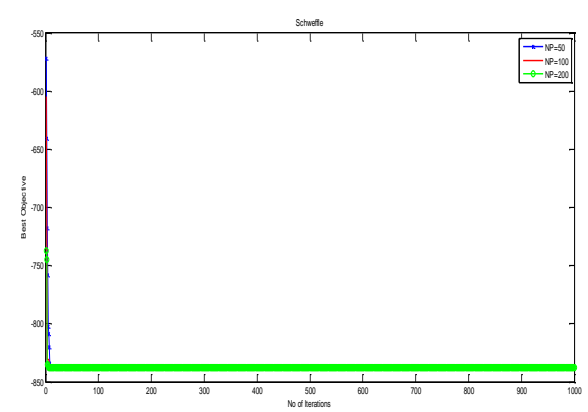

Fig. 4 Beale

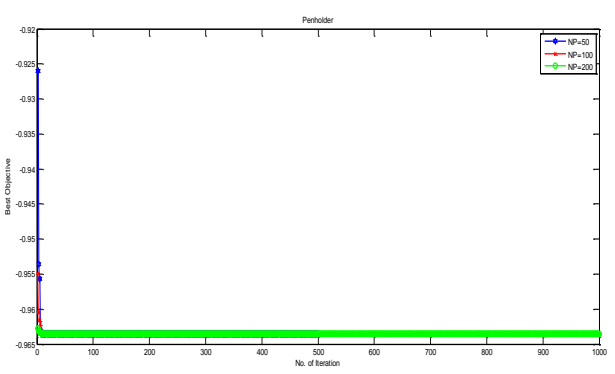

Fig. 9 Easom

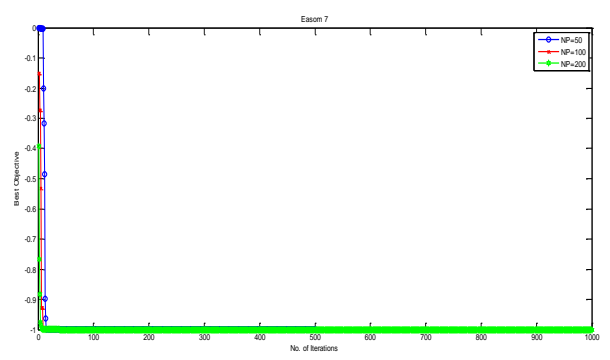

Fig. 10 Penholder

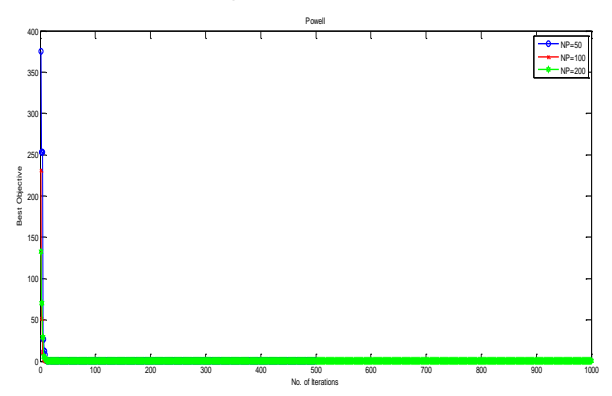

Fig. 11 Powell

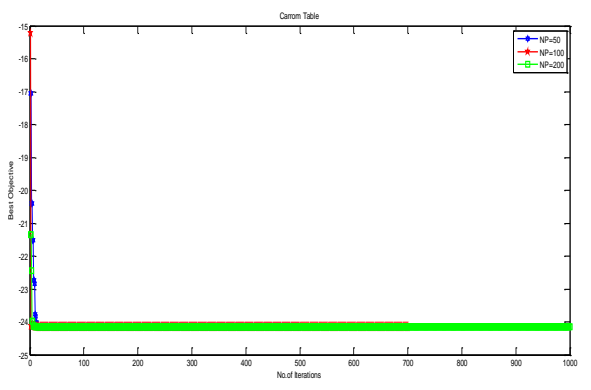

Fig. 12 Rastrigin 


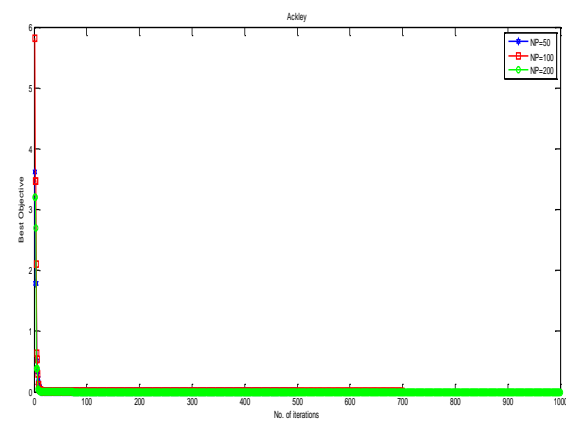

Fig. 13 Schweffel

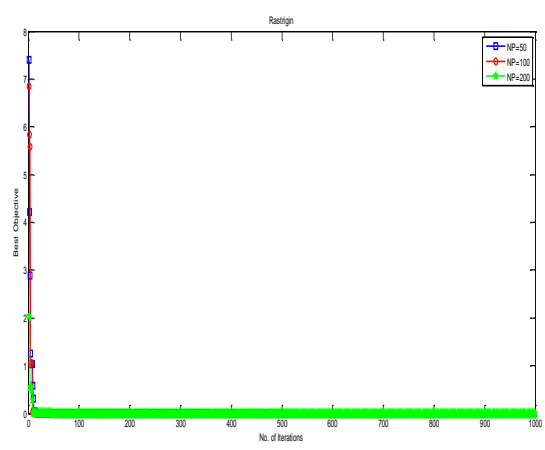

Fig. 14 Sphere

When we choose the following population size (NP) are $50,100,200$ corresponding to the No. of Iterations are100, 200, 300 respectively with the consideration of 12 Benchmark functions are performing divergence in the given range of interval. However we change the slight variation in population size (NP) are 50,100,200 corresponding to the No. of Iterations are 500, 700, 1000 respectively which performing on above said benchmark function then it provides very smoothly convergence.

\section{CONCLUSION}

In this investigation, The convergence rate of the various function such as Ackley, Beale, Booth, Carrom-table, Crowned-cross, Cross-in-tray, Easom, Penholder, Powell, Rastrigin, Schweffel, Sphere have been examined by BBO algorithm, it is observed that the all considered fitness function are converged suitably. Obtained computational results are very much near to the defined results of the problem.

\section{REFERENCES}

[1] R. Macarthur and E. Wilson, "The theory of Biogeography, Princeton, NJ: Princeton University”, Press. 1967.

[2] G. Guo and S. Yu, "The unified method analyzing convergence of genetic algorithms, Control Theory \& Application", Vol. 18, No. 3, pp. 443-446, 2001.

[3] D. Simon, "Biogeography-Based optimization", IEEE Transactions on Evolutionary Computation, Vol. 12, No. 6, pp. 702-713, 2008.

[4] H. Ma and D. Simon, "Blended Biogeography-Based optimization for constrained optimization", Engineering Application of Artificial Intelligence, Vol. 24, No. 6, pp. 517-525, 2010.

[5] H. Ma, "An analysis of the equilibrium of migration models for biogeography-based optimization", Information Sciences, Vol. 180, No. 18, pp. 3444-3464, 2010.

[6] D. Simon, R. Rick., E and Mehmet D. Dawei, "Analytical and numerical comparisons of biogeography-based optimization and genetic algorithms", Information Sciences, Vol. 181, No. 7, pp. 12241248,2011

[7] B. Ilhem, Amitava Chatterjee, S. Patrick and Mohamed AhmedNacer, "Biogeography-based optimization for constrained optimization problems", Computers \& Operations Research, Vol. 39, No. 12, pp. 3293-3304, 2012.

[8] H. Ma, X. Yong Ruan and Z. Xin Pan, "Handling multiple objective with Biogeography based optimization", International Journal of Automation and Computing, Vol. 9, No. 1, pp. 30-36, 2012.

[9] N. F. Hordri, S. S. Yuhaniz and D. Nasien, "A Comparison study of Biogeography based Optimization for Optimization problems", International Journal Advance Soft Computing Application, Vol. 5, No. 1, pp. 1-16, 2013

[10] Q. Feng, S. Liu, Q. Wu, G. Tang, H. Zhang and H. Chen, "Modified Biogeography-Based optimization with Local Search Mechanism", Journal of Applied Mathematics, pp. 1-24, 2013. [Online]. Available: http://dx.doi.org/10.1155/2013/960524.

[11] G. Guo, W. Lei and Wu, Qidi, "An analysis of the migration rates for biogeography-based optimization", Information Sciences, Vol. 254, pp. 111-140, 2014

[12] H. Ma., D. Simon and Minrui Fei, "On convergence of Biogeography-Based optimization for Binary Problems", Mathematical Problems in Engineering, pp. 1-11, 2014. [Online]. Available: http://dx.doi.org/10.1155/2014/147457

[13] E. M. Golafshani, "Introduction of Biogeography based programming as a new algorithm for solving problems", Vol. 270 pp.1-12, 2015

[14] G. Weian, W. Lei and Wu. Qidi, "Numerical comparisons of migration models for Multi-objective Biogeography-Based Optimization", Information Sciences, Vol. 328, pp. 302-320, 2016

[15] H. Ma and D. Simon, "Biogeography-Based Optimization:A10 Year Review", IEEE Transactions on Emerging Topics in Computational Intelligence, Vol. 1, No. 5, pp. 391-407, 2017

[16] Gholamreza Khademi, Hanieh Mohammadi and Dan Simon, "Hybrid invasive weed/biogeography-based optimization", Engineering Applications of Artificial Intelligence, Vol. 64, pp. 213$231,2017$. 